\title{
Instrumento de avaliação de quedas para idosos (IAQI): enfermeiro analisando vulnerabilidade e fragilidade
}

\author{
Assessmetinstumetfor eddely falls(IAQ): nursesandyüng winerabilityandfragility
}

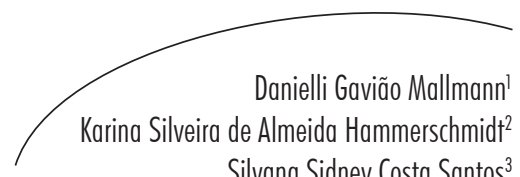

Resumo

Pesquisa qualitativa que teve por objetivo desenvolver instrumento de avaliação de quedas em idosos, a ser utilizado como apoio ao processo de enfermagem na Estratégia de Saúde da Família (ESF), considerando vulnerabilidade e fragilidade dessas pessoas para quedas. Realizou-se avaliação dos itens que compõem o protocolo de avaliação multidimensional do idoso, selecionando aqueles que se adequavam ao propósito do Instrumento de Avaliação de Quedas para Idosos (IAQI), criado neste estudo, e excluindo os que não possuíam relação direta com as quedas em idosos, argumentando sua exclusão. A análise dos dados ocorreu por meio da leitura dos itens do protocolo de avaliação multidimensional do idoso, identificando as particularidades clínicas relacionadas às quedas. Após a criação do IAQI, apresentou-se fluxograma expositivo de sua origem, tendo como itens selecionados para a composição do IAQI: identificação; queixa principal; revisão dos sistemas fisiológicos principais; avaliação da funcionalidade global; história pessoal atual e pregressa; avaliação ambiental; diagnósticos principais, contendo diagnósticos/problemas e diagnóstico funcional; plano de cuidados. O instrumento desenvolvido pode ser capaz de auxiliar na avaliação da vulnerabilidade e fragilidade do idoso para quedas, sendo indicado para embasar o roteiro da avaliação do enfermeiro que atua na ESF. O propósito final deste estudo foi proporcionar aos estudantes e professores a utilização de instrumento em suas práticas clínicas na ESF, visando ao melhor atendimento da pessoa idosa.

\section{Abstract}

Qualitative research aiming to develop an evaluation tool for falls in elderly, used as support for the nursing process in the Family Health Strategy (FHS), considering the vulnerability and fragility of these people for falls. We conducted evaluation of the items that make up the multidimensional assessment protocol for the elderly, selecting those

\footnotetext{
Curso de Pós-Graduação em Gestão Pública Municipal. Universidade Federal do Mato Grosso do Sul. Campo Grande, MS, Brasil.

2 Curso de Pós-Graduação em Enfermagem. Universidade Federal do Pampa. Uruguaiana, RS, Brasil.

Curso de Pós-Graduação em Enfermagem. Universidade Federal do Rio Grande. Rio Grande, RS, Brasil.
}

Palavras-chave: Idoso. Vulnerabilidade. Acidentes por quedas. Enfermagem geriátrica. Protocolos clínicos. 
that suited the purpose of the Assessment Instrument for Elderly Falls (IAQI), created within this research work, and excluding those who had no direct relation to the falls in the elderly, arguing their exclusion. Data was analyzed by reading the items in the multidimensional assessment protocol for the elderly, identifying the specific clinical findings related to falls. After the creation of IAQI, an expositive flowchart presented its origin, with the selected items to the composition of IAQI: identification, chief complaint, review of major physiological systems; assessment of global functionality, current and past personal history, environmental assessment, primary diagnoses, including diagnosis / problems and functional diagnosis, plan of care. This tool may help assessing the vulnerability and fragility of the elderly to falls, and be used to underscore the assessment of the nurse's who work in the FHS. The ultimate purpose of this study was to provide students and teachers, the possibility of using the instrument in their clinical practice in the the FHS, in order to better care of the elderly.
Key words: Aged. Vulnerability. Injuries from falls. Geriatric nursing. Clinical protocols.

\section{INTRODUÇÃO}

O envelhecimento da população idosa incita discussão sobre as necessidades de saúde que as pessoas idosas apresentam atualmente. Com o envelhecimento, ocorrem modificações biopsicossociais ${ }^{1}$ que podem interferir na saúde do idoso, por determinarem a perda da capacidade do indivíduo para se adaptar ao ambiente, podendo acarretar maior vulnerabilidade e incidência de processos patológicos, ${ }^{2,3}$ ocasionados pela fragilidade que pode surgir no processo de envelhecimento.

Entende-se fragilidade como síndrome clínica caracterizada por declínio progressivo de energia, relacionada às alterações nos sistemas muscular, neuro-endócrino e imunológico, podendo levar a vulnerabilidade dos fatores estressores e incapacidade de manter a homeostase nessas situações, com maior risco de eventos adversos. ${ }^{4}$ Como consequência da fragilidade, tem-se a vulnerabilidade, a qual é definida como conjunto de fatores biológicos, epidemiológicos, sociais e culturais cuja interação amplia o risco da pessoa em relação à doença, a certa condição ou a um dano, ${ }^{5}$ bem como às quedas.

A temática desta pesquisa, "quedas da pessoa idosa", mostra-se de importância primordial para a realidade brasileira, a qual vem apresentando alterações na estrutura da pirâmide etária desde a década de 1970, constatando-se o crescimento quantitativo da população idosa. Com esse crescimento, prevê-se que o Brasil será o sétimo país com maior número de pessoas idosas no ano de 2025, conforme dados divulgados no U. S. Bureau of Census de 1996. ${ }^{6}$

Algumas questões podem ser mais prevalentes em decorrência do processo de envelhecimento, dentre as quais se destacam os acidentes por quedas, que são ocasionados por diversos fatores, como alterações de equilíbrio, este preponderantemente o mais afetado, propiciando instabilidades e ocorrências de quedas. ${ }^{7}$ Estas apresentam alto índice de prevalência entre os idosos e aumentam com a idade, ${ }^{3,8}$ chegando a $32 \%$ nos idosos de 65 a 74 anos, e $51 \%$ nos idosos com mais de 85 anos. $^{8}$

A queda pode ser definida como deslocamento não-intencional do corpo resultando em mudança da posição para um nível inferior à posição inicial, com incapacidade de correção em tempo hábil, ${ }^{8}$ podendo ser causada por aspectos intrínsecos e extrínsecos. Entre os fatores predisponentes intrínsecos estão aqueles relacionados à saúde da pessoa, ou seja, de ordem interna. Já os fatores extrínsecos são relacionados com o ambiente em que a pessoa vive, ou seja, de ordem externa.

Entre os fatores associados às quedas, estão: polifarmácia, múltiplas patologias, comprometimento cognitivo, diminuição da acuidade visual, fraqueza óssea, dificuldade 
para deambulação e barreiras ambientais, ${ }^{3}$ como tapetes. A maioria das quedas ocorre durante as atividades rotineiras do idoso ${ }^{3}$ e gera o medo de cair novamente, que propicia redução das habilidades na realização das atividades de vida diária (AVD). E entre as consequências das quedas estão as fraturas, ${ }^{3}$ que podem acarretar dependência do idoso e sua institucionalização, gerando custos com os cuidados de saúde.

Após a ocorrência da queda, pode haver necessidade de encaminhar a pessoa idosa a atendimento de saúde da localidade ou região em que ela reside. Destaca-se o papel das unidades básicas que integram a Estratégia Saúde da Família (ESF), a qual é entendida como modelo de reorientação da Atenção Básica, desenvolvida por meio da implantação de equipes multiprofissionais em unidades básicas de saúde. ${ }^{?}$

$\mathrm{Na}$ ESF, a pessoa idosa é atendida por profissionais da saúde, em especial pelo enfermeiro, que, segundo o Caderno de Atenção Básica, Envelhecimento e Saúde da Pessoa Idosa, do Ministério da Saúde, ${ }^{10}$ tem como atribuição realizar consulta de enfermagem, incluindo a avaliação multidimensional do idoso. Nessa perspectiva, o Código de Ética dos Profissionais da Enfermagem dispõe que o enfermeiro é responsável pela avaliação clínica, bem como pela avaliação de informações, de forma a contemplar os aspectos que podem estar inseridos no processo de enfermagem. ${ }^{11}$

Percebe-se a relevância de um instrumento para avaliação da vulnerabilidade e fragilidade do idoso, ao se analisar o já citado Caderno de Atenção Básica, Envelhecimento e Saúde da Pessoa Idosa, do Ministério da Saúde, ${ }^{10}$ que recomenda o estabelecimento de critérios que identifiquem as pessoas idosas que se encontram fragilizadas ou em condição subclínica de fragilidade, a fim de evitar, adiar ou amenizar as respostas adversas a esta. Corroborando esta afirmação, autores $^{12}$ afirmam que a avaliação deve ser individualizada pelo fato de cada ser humano apresentar particularidades e especificidades biopsicológicas.
Após revisão da literatura para conhecimento da existência de instrumentos de avaliação das quedas em idosos, de uso do enfermeiro, e a constatação da inexistência de tal instrumento, percebeu-se a necessidade de se construir um instrumento para o processo de enfermagem, adaptado ao idoso, que considere a vulnerabilidade e a fragilidade. Tal instrumento poderá ser utilizado, pelo enfermeiro, na avaliação dos idosos que sofreram quedas, a fim de padronizar os itens e escalas utilizados na obtenção de resultados de suas atuais condições funcionais e promover a prevenção das quedas. Assim, o presente estudo tem como pergunta de pesquisa: como deveria ser constituído um instrumento para avaliação da vulnerabilidade e fragilidade do idoso caidor, a ser utilizado pelo enfermeiro que atua na ESF?

Diante do exposto, este estudo teve como objetivo desenvolver instrumento de avaliação de quedas para as pessoas idosas, a ser utilizado como apoio ao processo de enfermagem na Estratégia Saúde da Família, considerando vulnerabilidade e fragilidade desta população.

\section{METODOLOGIA}

Trata-se de pesquisa qualitativa, que envolve lógica, planejamento e meticulosidade nos métodos aplicados na coleta de dados, além de cuidado, ponderamento e rigor na análise dos dados coletados..$^{13} \mathrm{O}$ estudo foi realizado no período de agosto de 2009 a junho de 2010, compondo o trabalho de conclusão de curso de uma das autoras. É composto por três etapas: 1) avaliação dos itens que compõem o protocolo de avaliação multidimensional do idoso; ${ }^{14}$ 2) análise crítica dos itens envolvidos na avaliação da pessoa idosa que possuam relação com as quedas; 3) desenvolvimento do instrumento direcionado ao processo de enfermagem na Estratégia Saúde da Família (ESF), visando à avaliação da vulnerabilidade e fragilidade para quedas, elaborado com base nos itens analisados na etapa anterior.

$\mathrm{Na}$ primeira etapa, foram avaliados os itens que compõem o Protocolo de Avaliação 
Multidimensional do Idoso, ${ }^{14} \mathrm{com}$ o intuito de obter conhecimento e reflexão sobre cada item vinculando com as quedas em idosos. Posteriormente, foram selecionados os itens do protocolo que possuíam relação com as quedas, e excluídos os que não se adequavam a este propósito.

O Protocolo de Avaliação Multidimensional do Idoso $^{14}$ é composto por vários instrumentos que proporcionam diversas avaliações da pessoa idosa, tanto no âmbito da funcionalidade global quanto dos sistemas fisiológicos, além de apresentar avaliação do ambiente em que o idoso vive.

As avaliações que compõem o protocolo são: revisão dos sistemas fisiológicos principais; avaliação da funcionalidade global como atividades da vida diária, atividades instrumentais da vida diária, mobilidade, marcha e equilíbrio; avaliação da cognição; avaliação ambiental e diagnósticos principais. Com sua utilização, pode-se elaborar plano de cuidados, a fim de recuperar ou manter a independência e autonomia do idoso.

Durante a segunda etapa, foram analisados os itens de avaliação de idosos que apresentaram relação com as quedas fazendo análise crítica dos mesmos. Considerou-se a análise dos itens existentes no Protocolo de Avaliação Multidimensional do Idoso, ${ }^{14}$ elencando os motivos pelos quais se adequavam a este estudo. A análise foi realizada por meio da leitura dos itens que compunham esse protocolo, ${ }^{14}$ identificando as particularidades relacionadas às quedas.

Para atender à terceira etapa do estudo, foram reunidos os itens de avaliação da pessoa idosa, relacionados com as quedas, para formulação e desenvolvimento de instrumento para o processo de enfermagem direcionado aos idosos cadastrados/atendidos na ESF, visando à avaliação da vulnerabilidade e fragilidade para quedas. Este instrumento é voltado para ação de cuidar dos enfermeiros, com aplicabilidade durante a etapa de investigação do processo de enfermagem. Foram selecionados os itens que poderiam ser aplicados por esses profissionais e nenhum foi alterado ou modificado, respeitandose a validação do protocolo, já realizada. ${ }^{14}$

A validação do Instrumento de Avaliação de Quedas em Idosos será realizada em estudo posterior, com apresentação do instrumento para utilização por enfermeiros da ESF, visando a apurar possíveis problemas na sua aplicação.

Para a realização do estudo, foram utilizadas fontes secundárias, ou seja, de materiais de domínio público, e foram consideradas as normas nacionais e internacionais sobre direitos autorais dos estudos analisados. Respeitaramse as citações de fontes, bem como a devida apresentação dos autores quando mencionada a produção realizada por estes.

\section{RESULTADOS E DISCUSSÃO}

O instrumento criado neste estudo é composto, primeiramente, pelo item identificação, é imprescindível para o Instrumento de Avaliação de Quedas para Idosos (IAQI), pois a identificação é o ponto de partida para o conhecimento da pessoa idosa e de sua família. ${ }^{15}$ Com este item, pode-se ter pistas sobre a vulnerabilidade e fragilidade do idoso, por questionar idade, sexo e estado civil, os quais interferem diretamente na avaliação da pessoa idosa. A idade superior a 80 anos significa maior grau de vulnerabilidade; já se for mulher, a pessoa idosa pode apresentar maior risco de fragilização e dependência funcional; e o estado civil pode indicar o risco de fragilização do idoso caso este apresente viuvez. ${ }^{15}$

O próximo item a compor o instrumento é a queixa principal, pois nas avaliações da pessoa idosa deve-se saber a queixa do idoso no momento da consulta, embora as condições associadas ao envelhecimento, como as quedas, não sejam a queixa principal, mas podem estar associadas a ela. ${ }^{15}$

Outros itens pertinentes ao instrumento compõem a revisão dos sistemas fisiológicos principais, 
sendo a pele e anexos o primeiro, o qual se adequa ao IAQI por avaliar a presença de lesões na pele, ${ }^{15}$ as quais podem prejudicar o caminhar do idoso, podendo levá-lo a episódios de quedas.

A avaliação do aparelho cardiovascular, próximo item selecionado, mantém-se no instrumento proposto por investigar aspectos como hipotensão ortostática e síncope, ${ }^{15}$ os quais podem ser fatores de risco para quedas. ${ }^{16}$ Hipotensão ortostática significa queda da pressão arterial sistólica superior ou igual a 20 $\mathrm{mmHg}$ e/ou queda da pressão arterial diastólica maior ou igual a $10 \mathrm{mmHg}$, no momento em que a posição de decúbito é modificada para a posição ortostática, ou ainda após três minutos dessa movimentação..$^{17,18}$ Pode ocasionar tonturas, tremores, dor de cabeça, quedas, síncope, entre outras consequências. ${ }^{18}$ Síncope é definida como a perda repentina do nível de consciência e do tônus postural, seguida de recuperação espontânea e imediata. ${ }^{19}$

Outro item selecionado diz respeito aos órgãos dos sentidos, pois investiga se a pessoa idosa apresenta deficiência visual, vertigem, entre outros aspectos ${ }^{15}$ que podem ocasionar quedas. ${ }^{16}$ A deficiência visual prejudica a locomoção do idoso nos ambientes, por não visualizar os objetos que estão em seu caminho, e a vertigem é a sensação de movimento rotatório, normalmente ocasionada por desordens no sistema vestibular. ${ }^{16,20}$

Da mesma maneira, a avaliação do aparelho geniturinário fará parte do IAQI, por investigar a presença de redução da habilidade de adiar a micção e incontinência urinária. ${ }^{15}$ Problemas relacionados à micção podem ser fatores predisponentes das quedas nas pessoas idosas, por impor deslocações rápidas ao banheiro. ${ }^{21}$ Este fato incita a maior probabilidade da ocorrência de quedas, sobretudo no período noturno e em ambientes com baixa luminosidade.

O IAQI apresentará também o item sistema músculo-esquelético, que avalia fraqueza muscular, artrite, rigidez, afecções dos pés, entre outros aspectos, ${ }^{15}$ que podem ocasionar quedas. ${ }^{21,22}$
A avaliação do aparelho digestivo mantevese no instrumento por investigar a presença de incontinência fecal, ${ }^{15}$ visto que os idosos que apresentam maior risco de incontinência são aqueles com demência, depressão grave e instabilidade postural/imobilidade. ${ }^{23} \mathrm{~A}$ presença de incontinência fecal pode ser indicativa de instabilidade postural, fator determinante na ocorrência de quedas nas pessoas idosas.

Ainda dentro da revisão dos sistemas fisiológicos principais, está a avaliação do sistema nervoso, que investiga presença de redução da força muscular e tonturas, ${ }^{15}$ aspectos diretamente relacionados a quedas, pois a fraqueza muscular causa prejuízo locomotor, retardando as reações de equilíbrio, sendo importante determinante do índice de quedas, ${ }^{24}$ e indica a hipótese de causaefeito entre a força muscular e as quedas. ${ }^{25}$

Outra possível causa de quedas em idosos podem ser as tonturas. A tontura é sensação de mudança do equilíbrio corporal, podendo ser consequência de alterações funcionais primárias ou secundárias do sistema vestibular. ${ }^{20}$ Diante dos aspectos avaliados neste item, a tontura foi selecionada para compor o IAQI, por se adequar aos seus propósitos.

Além dos itens descritos, a avaliação da funcionalidade global é pertinente ao instrumento deste estudo, pois avalia a autonomia e independência da pessoa idosa para realizar as atividades de vida diária, as quais contribuem para manutenção do bem-estar e da qualidade de vida do idoso. ${ }^{15}$

Um item que compõe a avaliação da funcionalidade global é a avaliação das atividades de vida diária básicas, presente no IAQI, pois estima o comprometimento funcional, bem como o grau de dependência do idoso, ${ }^{15}$ sendo composta pela escala de Katz, que avalia o desempenho da pessoa nas atividades de autocuidado. ${ }^{26} \mathrm{~A}$ escala de Katz examina desde atividades simples, consideradas vegetativas - transferência, continência e alimentação -, até as atividades mais complexas, como banhar-se, vestir-se e usar o banheiro. ${ }^{15}$ 
Com esta análise, a pessoa idosa pode ser categorizada como independente, sendo aquele que realiza todas as atividades básicas da vida diária independentemente. Semidependente é o idoso que apresenta comprometimento de uma das funções que são influenciadas pelo aprendizado e cultura. $\mathrm{O}$ dependente incompleto apresenta comprometimento de alguma função vegetativa simples, como tomar banho, vestirse e usar banheiro. E o dependente completo apresenta dependência para a realização de todas as atividades básicas da vida diária. ${ }^{15}$

O comprometimento funcional das atividades da vida diária pode indicar alguma doença não tratada que não apresentou sintomas típicos, não devendo ser atribuída ao envelhecimento a perda de alguma função previamente independente. ${ }^{15}$ As atividades da vida diária são influenciadas pela mobilidade, que é essencial para a execução plena das mesmas, e está diretamente ligada a fatores intrínsecos e extrínsecos, ${ }^{27}$ podendo, em casos de comprometimento, ocasionar quedas ${ }^{28}$ e, portanto, a avaliação da mobilidade permanece como item relevante do IAQI.

Entre os fatores ligados à mobilidade está a postura/marcha, que depende do equilíbrio da pessoa, ${ }^{27}$ podendo ser este fator importante para a ocorrência de quedas em idosos. ${ }^{29}$ A avaliação quantitativa e qualitativa da marcha e equilíbrio, item indispensável ao IAQI, deve compor este instrumento por permitir o reconhecimento da presença de instabilidade postural ou alteração da marcha, ${ }^{27}$ que são fatores de risco de quedas, podendo indicar anormalidades músculoesqueléticas e/ou neurológicas. ${ }^{16}$

Além dos achados sobre marcha e equilíbrio, a importância de tal item para o IAQI também é vista no questionamento sobre a história de quedas no último ano, pois este é fator relevante para prever futuras quedas, ${ }^{30}$ visto que é necessária a prevenção da primeira queda ou de quedas repetitivas.

Já o item cognição apresenta dois testes selecionados para compor o IAQI, sendo Mini-
Mental e Clinical Dementia Rating (CDR). O MiniMental é utilizado para a triagem de idosos com déficit cognitivo, avaliação da evolução de demenciados, bem como para o monitoramento de resposta terapêutica. ${ }^{31}$ Avalia a memória de curto prazo, bem como a memória episódica e semântica. A memória de curto prazo é dividida em memória de trabalho e memória imediata, sendo entendida como a capacidade de guardar informações limitadas por alguns segundos. ${ }^{31}$

Este item avalia a linguagem e gnosia. ${ }^{31}$ A gnosia é a capacidade de decodificação e reconhecimento de estímulos do mundo exterior, podendo ser visual, auditiva, tátil ou associativa, ${ }^{31}$ sendo, portanto, a capacidade de perceber e reconhecer os objetos por meio de um dos sentidos. Assim, após o reconhecimento, pode-se agir conforme os estímulos recebidos do meio exterior, e caso haja comprometimento dessa função, pode haver prejuízo nas realizações de atividades da vida diária, o que pode ocasionar quedas.

O Mini-Mental também avalia a praxia, função executiva e função visuoespacial. ${ }^{31}$ Devido aos aspectos avaliados por esse teste, ele foi selecionado por se adequar ao propósito do IAQI, criado neste estudo.

A escala Clinical Dementia Rating (CDR) é direcionada aos domínios $\operatorname{cognitivos}^{31}$ e se mantém no presente estudo por ter a capacidade de integrar aspectos de ordem cognitiva e comportamental, predizendo o quanto interferem nas atividades de vida diária, ${ }^{32}$ as quais devem ser entendidas como aspectos importantes na identificação da vulnerabilidade e fragilidade que a pessoa idosa possui para quedas. E para que a avaliação da fragilidade e vulnerabilidade para quedas nos idosos seja adequada, deve-se conhecer sua historia pessoal atual e pregressa, pois por meio dela é possível descobrir o uso de medicamentos, prática de atividade física, uso de próteses/órteses, doenças diagnosticadas anteriormente, como parkinsonismo, acidente vascular encefálico (AVE), osteoporose, catarata, entre outros aspectos que podem indicar risco para quedas. 
A diminuição da prática de atividade física torna a pessoa idosa propensa a quedas, pois diminui a independência e autonomia que ela possui para realizar as atividades de vida diária. ${ }^{28,33}$ Doenças como Parkinson podem contribuir para a ocorrência de quedas, corroborando o estudo $^{33,34}$ que indica que doenças neurológicas como Parkinson e AVE podem ser fatores de risco para quedas. Além destes fatores, doenças degenerativas das articulações, diabetes mellitus, problemas nos pés e distúrbios da visão e audição também podem ocasionar quedas. ${ }^{22}$

As quedas das pessoas idosas podem ter causas multifatoriais, entre as quais se destaca a inadequação ambiental, pois o ambiente pode ser barreira para a independência do idoso nas atividades cotidianas. ${ }^{35}$ Assim, a avaliação ambiental é pertinente a este estudo, por apresentar um questionário domiciliar sobre os riscos para quedas que o ambiente pode apresentar. Percebese que tal item é essencial ao IAQI pelo fato dos fatores causais das quedas, relacionados ao ambiente, serem responsáveis por sua maior incidência, afirmando que a ausência de adequações no ambiente, como iluminação, piso adequado e barras de apoio, são responsáveis por $44 \%$ das quedas em idosos. ${ }^{22}$

Ao finalizar a avaliação da pessoa idosa, é indispensável que se faça diagnóstico funcional, pois este avalia o comprometimento funcional do idoso, incluindo as atividades da vida diária, cognição, humor, mobilidade, comunicação, interação social e outras funções orgânicas, ${ }^{15}$ resumindo então os testes aplicados na avaliação da pessoa idosa.

$\mathrm{O}$ item diagnósticos/problemas especificos, selecionado para compor o IAQI, serve como complementação do diagnóstico funcional, trazendo espaço para descrição dos problemas observados nos sistemas fisiológicos principais, que têm relevância para a saúde do idoso.

Finalizando a seleção de itens a compor o IAQI, tem-se o item plano de cuidados. Diante do resultado obtido com o IAQI, é fundamental delinear o plano de cuidados para a pessoa idosa, propondo ações de promoção da saúde e prevenção de doenças, tratamento dos problemas observados durante a avaliação, reabilitação para incapacidades observadas, podendo ser atribuídas ações que previnam ou estabilizem o comprometimento funcional do idoso.

Consideração importante deve ser dada ao enfermeiro, que pode utilizar o plano de cuidados, realizando, além de observações e orientações, dados não inclusos em outras etapas do processo de avaliação. ${ }^{15}$ Sendo assim, o IAQI deve contemplar, além de todos os itens selecionados anteriormente, o plano de cuidados. 


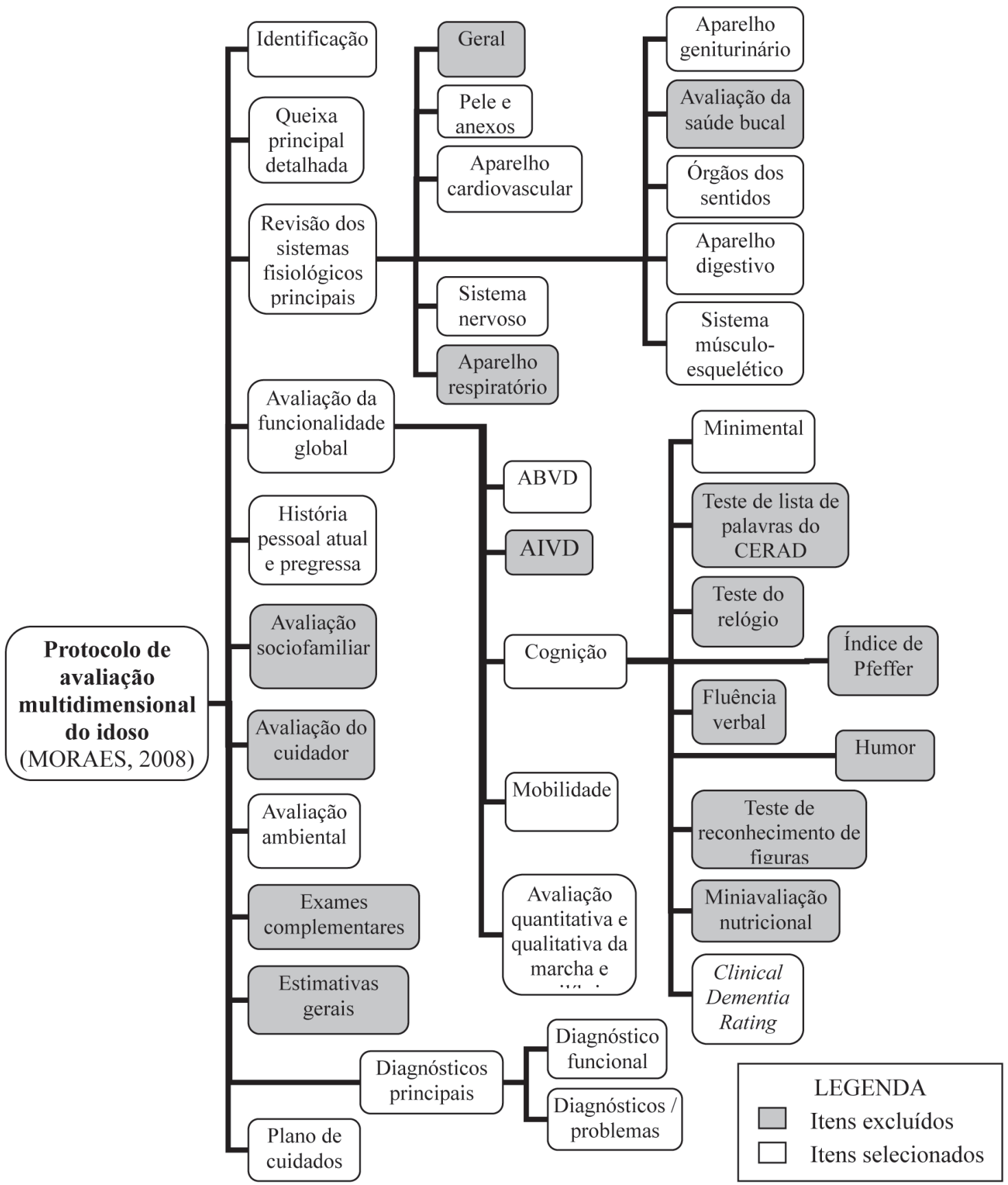

Figura 1 - Instrumento de Avaliação de Quedas em Idosos (IAQI): Composição; Fluxograma visual da construção do IAQI. 


\section{CONSIDERAÇÕES FINAIS}

Com base neste estudo, pode-se desenvolver instrumento capaz de auxiliar na avaliação da vulnerabilidade e fragilidade da pessoa idosa para quedas, sendo indicado para embasar o roteiro da avaliação do enfermeiro que atua na Estratégia de Saúde da Família (ESF) atingindo o objetivo proposto neste estudo.

O Instrumento de Avaliação de Quedas em Idosos (IAQI) poderá possibilitar o planejamento de ações de cuidado de enfermagem relacionadas às pessoas idosas, bem como pode direcionar o processo de enfermagem para essa população. Tais afirmativas se devem à dimensão da problemática das quedas e à abrangência das ações de enfermagem frente à necessidade de cuidados requeridos pelo idoso.

Com a utilização do IAQI, poderá haver melhoria da prática prestada e, consequentemente, benefícios para as pessoas idosas que sofreram quedas, pois terão uma avaliação com um roteiro que as auxiliará na completude da investigação clínica da vulnerabilidade e fragilidade para quedas, podendo prevenir possíveis quedas futuras.

O IAQI tende a repercutir positivamente no cotidiano de trabalho dos enfermeiros, pois, ao realizarem a consulta de enfermagem, terão instrumento apropriado para a avaliação da vulnerabilidade e fragilidade das pessoas idosas para quedas, indispensável para sua prevenção.

Do mesmo modo, as pessoas idosas serão beneficiadas com o IAQI, pois a partir de sua utilização na investigação do processo de enfermagem serão observados aspectos que podem ocasionar quedas para os idosos, e com base nos resultados será elaborado plano de cuidados objetivando prevenir possíveis danos à saúde do idoso, sobretudo quedas.

Este estudo proporcionará, aos estudantes, professores e enfermeiros, possibilidade de utilização de instrumento em suas práticas clínicas na ESF, visando ao melhor atendimento à pessoa idosa. E para o ensino em Enfermagem, o IAQI pode ser utilizado para ampliar os recursos no campo prático, a fim de aplicá-lo como instrumento de avaliação. Para a pesquisa, amplia as possibilidades de estudos relacionados a pessoas idosas e, para a extensão, cria meios para socialização do IAQI com idosos, familiares e profissionais.

O IAQI tem sua utilização voltada para a ESF por esta possuir protocolo nacional editado pelo Ministério da Saúde, que recomenda a realização da consulta de enfermagem, juntamente com a avaliação da pessoa idosa e atuações na comunidade, sendo essas competências do profissional enfermeiro desse serviço. Foi desenvolvido principalmente para o enfermeiro da ESF, pois este pode agir envolvendo características do domicílio do idoso, por meio das visitas domiciliares. Com as visitas, o enfermeiro da ESF pode realizar a avaliação ambiental, aspecto de grande relevância para a detecção de fatores de risco para quedas das pessoas idosas, pois a inadequação do ambiente em que o idoso vive é considerada a principal causa de queda.

Considerando o IAQI como adaptação do Protocolo de Avaliação Multidimensional do Idoso, torna-se imprescindível salientar que este foi escolhido devido à possibilidade de ser utilizado por equipe multidisciplinar, incluído o enfermeiro. Optou-se por este protocolo pelo embasamento teórico que possui, além de ser composto por testes conhecidos e validados.

O estudo apresentou como limitação de pesquisa o curto prazo para a sua elaboração, não podendo ser aplicado nas práticas clínicas do curso de Enfermagem. Em contrapartida, apresenta como fortaleza sua especificidade na avaliação da vulnerabilidade e fragilidade da pessoa idosa para quedas, criando instrumento único para ser utilizado pelo profissional enfermeiro da ESF. 


\section{REFERÊNCIAS}

1. Vieira EB. Manual de gerontologia: Um guia teóricoprático para profissionais, cuidadores e familiares. 2 ed. Rio de Janeiro: Revinter; 2004.

2. Papaléo Netto M. Processo de envelhecimento e longevidade. In: Papaléo Netto M. Tratado de Gerontologia. 2 ed. São Paulo: Atheneu; 2007. p. 3-14.

3. Santos MLC, Andrade MC. Incidência de quedas relacionada aos fatores de riscos em idosos institucionalizados. Rev Baiana de Saúde Pública 2005 jan-jun; 29(1): 57-68.

4. Fried LP, et al. Frailty in older adults: Evidence for a phenotype. Journal of Gerontology: Medical Sciences 2001; 56(3): 146-156.

5. Asinelli-Luz A, Fernades Júnior N. Gênero, adolescências e prevenção ao HIV/AIDS. ProPosições 2008; 19(2): 81-97.

6. Paschoal SMP, Franco RP, Salles RFN. Epidemiologia do envelhecimento. In: Papaléo Netto M. Tratado de Gerontologia. 2 ed. São Paulo: Atheneu; 2007. p. 39-56.

7. Baloh RW., Ying SH, Jacobson KM. A longitudinal study of gait and balance dysfunction in normal older people. Arch Neurol 2003 jun; 60(6): 835-39.

8. Pereira SRM, Burksman S, Perracini M, Py L, Barreto KML, Leite VMM. Quedas em idosos. Sociedade Brasileira de Geriatria e Gerontologia 2001 jun: $1-8$

9. Brasil. Ministério da Saúde. Secretaria de Atenção à Saúde. Departamento de Atenção Básica. Política Nacional de Atenção Básica. 4 ed. Brasília: Ministério da Saúde; 2007.

10. Brasil. Ministério da Saúde. Secretaria de Atenção à Saúde. Departamento de Atenção Básica. Envelhecimento e Saúde da Pessoa Idosa. Brasília: Ministério da Saúde; 2006.

11. Conselho Federal de Enfermagem. Resolução no 160, de 12 de maio de 1993. Aprova o Código de Ética dos Profissionais de Enfermagem. Rio de Janeiro: 1993. [Acesso em 28 jun 2010]. Disponível em: URL: http://pnass.datasus.gov.br/documentos/ normas/109.pdf

12. Mallmann DG, et al. Produção científica sobre o idoso e quedas: revisão integrativa. In: Santos SSC, Pelzer MT, Barros EJL, organizadoras. Quedas em idosos: reflexões a partir de produções científicas da Enfermagem da FURG. Rio Grande: FURG; 2010. p. $67-75$
13. Pope C, Mays N. Pesquisa qualitativa na atenção à saúde. 3 ed. Porto Alegre: Artmed; 2009.

14. Moraes EN. Protocolo de avaliação multidimensional do idoso. In: Moraes EN. Princípios básicos de Geriatria e Gerontologia. Minas Gerais: COOPMED; 2008. p. 157-88.

15. Moraes EN, et al. Avaliação clínico-funcional do idoso. In: Moraes EN. Princípios básicos de Geriatria e Gerontologia. Minas Gerais: COOPMED; 2008. p. 63-84.

16. Megale RZ, Moraes EN. Instabilidade postural: abordagem diagnóstica. In: Moraes EN. Princípios básicos de Geriatria e Gerontologia. Minas Gerais: COOPMED; 2008. p. 381-94.

17. Ducla-Soares JL. Hipotensão ortostática: o estado da arte. Medicina interna 2001; 8(2): 80-8.

18. Lopes LS, et al. Hipotensão ortostática em pacientes idosos ambulatoriais. Arq Med ABC 2007; 32(1): 17-20.

19. Benchimol M, Souza RO. Utilidade diagnóstica da massagem do seio carotídeo na posição ortostática durante o "Tilt Test". Arq Neuro-Psiquiatr 2003; 61(1): 87-90.

20. Tiensoli LO, Couto ER, Mitre EI. Fatores associados à vertigem ou tontura em indivíduos com exame vestibular normal. Rev CEFAC 2004; 6(1): 94-100.

21. Fabricio SCC, Rodrigues RAP, Costa Júnior ML. Quedas acidentais em idosos institucionalizados. Acta Paul Enf 2002 jul-set; 15(3): 51-9.

22. Barbosa MLJ, Nascimento EFA. Incidências de internações de idosos por motivo de quedas, em um hospital geral de Taubaté. Rev Biociênc 2001; 7(1): $35-42$.

23. Moraes EM, Meira MA. Incontinência fecal. In: Moraes EN. Princípios básicos de Geriatria e Gerontologia. Minas Gerais: COOPMED; 2008. p. 439-442.

24. Rebelatto JR, Castro AP, Chan A. Quedas em idosos institucionalizados: características gerais, fatores determinantes e relações com a força de preensão manual. Acta Ortop Bras 2007; 15(3): 151-54.

25. Carvalho J, Soares JMC. Envelhecimento e força muscular - breve revisão. Rev Port Cien Desp 2004; 4(3): 79-93.

26. Lino VTS, Pereira SRM, Camacho LAB, Ribeiro Filho ST, Buksman S. Adaptação transcultural da Escala de Independência em Atividades da vida diária (Escala de Katz). Cad Saúde Pública 2008; 24(1): 103-12. 
27. Moraes EM, Megale RZ. Avaliação da mobilidade. In: Moraes EN. Princípios básicos de Geriatria e Gerontologia. Minas Gerais: COOPMED; 2008. p. 105-114.

28. Guimarães LHCT, et al. Comparação da propensão de quedas entre idosos que praticam atividade física e idosos sedentários. Rev Neurociênc 2004; 12(2): 68-72.

29. Maciel ACC, Guerra RO. Prevalência e fatores associados ao déficit de equilíbrio em idosos. $\mathrm{R}$ bras Ci e Mov 2005; 13(1): 37-44.

30. Tromp AM, et al. Predictors for falls and fractures in the Longitudinal Aging Study Amsterdam. Journal of bone and mineral research 1998; 13(12): 1932-39.

31. Moraes EM, Lanna FGJS. Avaliação da cognição e do humor. In: Moraes EN. Princípios básicos de Geriatria e Gerontologia. Minas Gerais: COOPMED; 2008. p. 85-104.
32. Montano MBMM, Ramos LR. Validade da versão em português da Clinical dementia rating. Rev Saude Publ 2005; 39(6): 912-17.

33. Soares AV, et al. Estudo comparativo sobre a propensão de quedas em idosos institucionalizados e não-institucionalizados através do nível de mobilidade funcional. Fisioter Bras 2003; 4(1): 13-7.

34. Melo EG, Azevedo E. O reumatologista revisita as: Quedas no idoso. Temas de reumatologia clínica 2007; 8(4).

35. Vidigal MJM, Cassiano JG. Adaptação ambiental. In: Moraes EN. Princípios básicos de Geriatria e Gerontologia. Minas Gerais: COOPMED; 2008. p.125-138.

Recebido: 01/11/2011

Revisado: 25/4/2012

Aprovado: 30/4/2012 